\title{
Long-Term Follow-Up of 4 Patients with Conjunctival Amyloidosis
}

\author{
Alisa J. Prager ${ }^{a}$ Larissa A. Habib ${ }^{a}$ Talita Gambogi ${ }^{a} \quad$ Klaus J. Busam ${ }^{b}$ \\ Brian P. Marr ${ }^{\mathrm{a}}$ \\ ${ }^{a}$ Department of Ophthalmology, Memorial Sloan Kettering Cancer Center, New York, NY, USA; \\ ${ }^{b}$ Department of Pathology, Memorial Sloan Kettering Cancer Center, New York, NY, USA
}

\section{Established Facts}

- Amyloidosis can be classified as localized (1 body organ involved) or systemic (multiple organs involved).

- Conjunctival amyloidosis is rare and most commonly localized.

\section{Novel Insights}

- Most cases can be managed with local excision, but patients should be monitored for recurrent symptoms.

- Workup for systemic rheumatologic diseases and blood dyscrasias should be tailored to patients' clinical characteristics and symptoms.

\section{Keywords}

Conjunctival amyloidosis · Ocular amyloidosis · Treatment

\section{Abstract}

Conjunctival amyloidosis is a rare cause of ocular inflammation, mass, and hemorrhage that can be difficult to diagnose and treat. In this case series, we describe 4 patients with a histopathological diagnosis of conjunctival amyloidosis treated at a single institution. All patients underwent surgical excision and biopsy. On histopathological examination, 3 patients had local deposition of either kappa or lambda monoclonal immunoglobulin light chains, favoring localized amyloid light-chain amyloidosis. Systemic workup to exclude rheumatologic disorders (e.g., anti-neutrophil cytoplasmic antibody and rheumatoid factors) and hematological disorders (e.g., imaging, biopsies, and serum protein electrophoresis/urine protein electrophoresis) was negative except for a positive abdominal fat biopsy in 1 patient. Patients were followed for an average of 6.1 years (range 4 months to 15 years) with stable ocular disease.

\section{KARGER}

(c) 2018 S. Karger AG, Basel

E-Mail karger@karger.com

www.karger.com/oop
Brian P. Marr, MD

Columbia University Department of Ophthalmology

635 West 165th Street

New York, NY 10032 (USA)

E-Mail Bpm2133@cumc.columbia.edu 

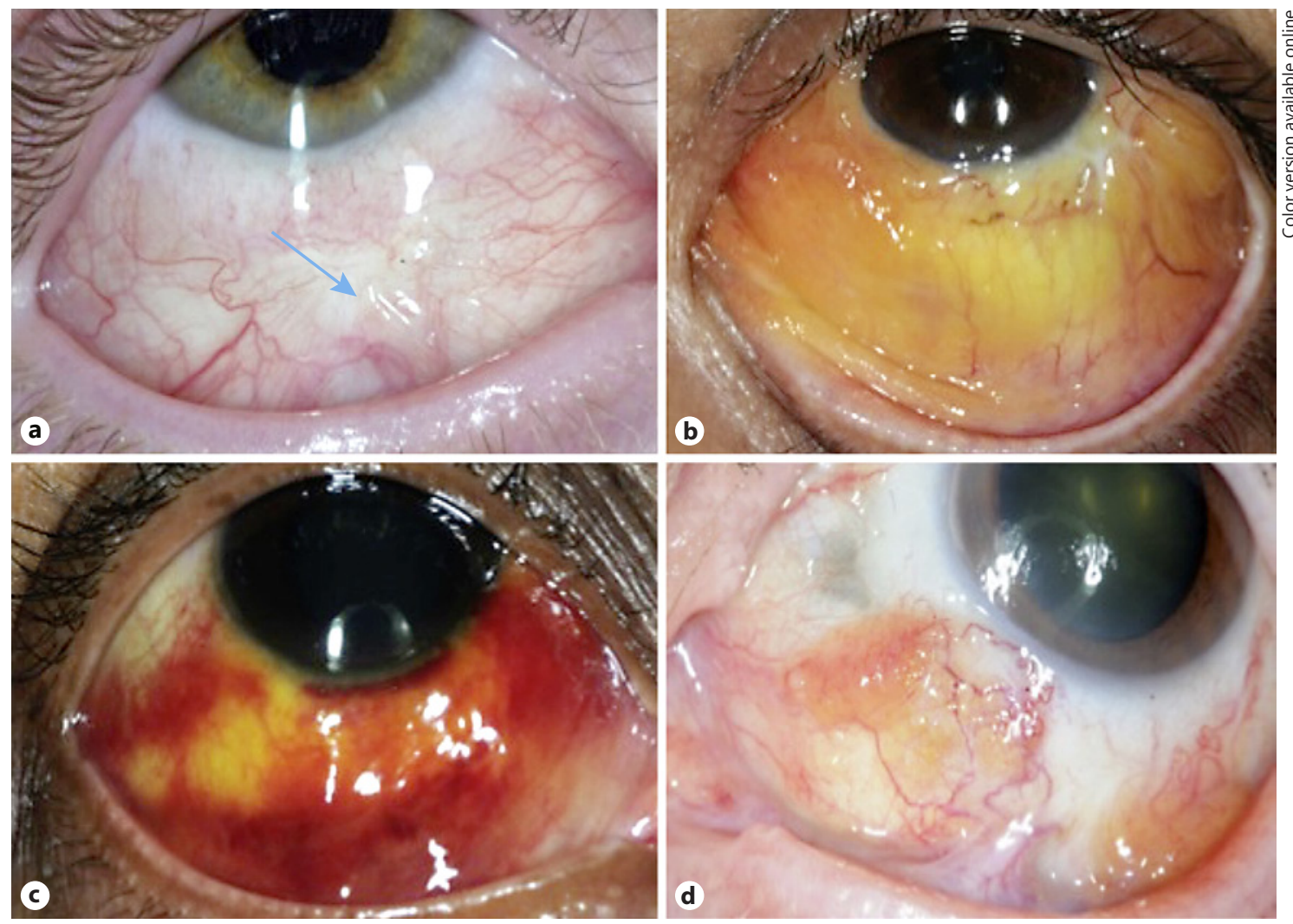

Fig. 1. Photographs of external eyes of the 4 patients showing a pinkish smooth mass with prominence of superficial blood vessels (arrow) (a), a diffuse yellowish-pink mass with prominent vessels (b), a yellowish waxy lesion with diffuse conjunctival hemorrhage (c), and a nodular yellow/salmon-colored mass with dilated vessels (d).

\section{Introduction}

Amyloidosis is characterized by extracellular deposition of abnormally folded, insoluble protein fibrils with $\beta$-sheet structure [1]. These fibrils are resistant to proteolysis and can disrupt the normal function of tissues, leading to severe organ dysfunction $[2,3]$. Amyloidosis is classified as either systemic, which can involve multiple organs, or localized, in which only 1 body organ is involved. The most common types of systemic amyloidosis are immunoglobulin amyloid light-chain (AL) amyloidosis and amyloid A (AA) amyloidosis.

Conjunctival amyloidosis is a relatively rare disease described in small case series and case reports [4-16]. There have been few reports of localized AL amyloidosis of the conjunctiva associated with no or trace systemic involvement $[15,17]$. However, conjunctival amyloidosis may be the initial sign of systemic disease [17-20], and systemic workup is recommended. This report describes the long-term follow-up of 4 cases with histologically proven conjunctival amyloidosis treated at a single institution.

\section{Case 1}

An 11-year-old Caucasian boy with an unremarkable medical history presented to an outside clinic with a painless red eye for 6 months. He was treated with fluorometholone and Patanol eye drops with minimal improvement. He was then referred to our institution for a second opinion. His best-corrected visual acuity (BCVA) was 20/25 in each eye, the intraocular pressure was 16 $\mathrm{mm} \mathrm{Hg}$ bilaterally, and extraocular movements were full. Slitlamp examination was notable for a smooth, pinkish conjunctival lesion in the right eye with prominent blood vessels (Fig. 1a). Dilated fundoscopic exam was unremarkable, and systemic workup for rheumatologic diseases was negative.

To establish a diagnosis, biopsy of the bulbar conjunctiva was performed. Immunohistochemical analysis of the specimen with 
Congo red stain was positive. There was also extracellular kappa light-chain deposition associated with kappa light-chain-restricted plasma cells. Systemic workup was negative for infectious studies (Epstein-Barr virus, hepatitis $\mathrm{C}$ virus, cytomegalovirus, HIV, hepatitis B virus, and Lyme disease) and rheumatologic studies (erythrocyte sedimentation rate, antinuclear antibody, anti-neutrophil cytoplasmic antibody, anti-SSA, anti-SSB, and anti-dsDNA). Furthermore, peripheral blood flow cytometry, serum protein electrophoresis, serum free light chains, immunofixation, and B-cell clonality studies of the conjunctiva and peripheral blood were negative. The family declined a bone marrow biopsy. He underwent a PET scan, which was unremarkable except for non-fluorodeoxyglucose-avid soft tissue superficial to the right eyeball. Cryotherapy was performed to treat the remaining lesion. The patient was followed for 4 years with no recurrence or growth of the lesion.

\section{Case 2}

A 31-year-old man with no significant past medical history presented to an outside clinic with a progressive, erythematous conjunctival lesion in his left eye. His BCVA was 20/20 in each eye, intraocular pressure was $14 \mathrm{~mm} \mathrm{Hg}$ bilaterally, and extraocular movements were intact. Fundoscopic exam was unremarkable. He underwent biopsy of the lesion, which showed amorphous eosinophilic, congophilic, and apple-green birefringent deposits in the substantia propria. He was diagnosed with conjunctival amyloidosis, and systemic workup was negative.

He continued to have recurrent episodes of conjunctival hemorrhage for 4 years and came to our institution for a second opinion. Examination was notable for a diffuse, smooth yellowishpink mass in the lower bulbar conjunctiva of the left eye with hyperemic vessels (Fig. 1b). Fundoscopic exam was negative. The lesion was observed for several months but remained symptomatic. He subsequently underwent excision and cryotherapy of the lesion. The biopsy showed amyloid deposits with Congo red stain and a small population of monotypic plasma cells with lambda predominance, consistent with the previous diagnosis of amyloidosis. He was followed for 5 years with intermittent recurrent episodes of conjunctival amyloidosis, which were observed. A bone marrow biopsy was done, which ruled out systemic amyloidosis and hematological malignancies.

\section{Case 3}

A 54-year-old African American woman with no significant past medical history presented to our clinic with multiple episodes of recurrent, worsening subconjunctival hemorrhage and tearing in her right eye for 8 months. Her BCVA was 20/20 in each eye, intraocular pressure was $11 \mathrm{~mm} \mathrm{Hg}$ bilaterally, and extraocular movements were full. Slit-lamp exam was notable for a diffuse, hyperemic, and hemorrhagic inferior conjunctival mass with yellowish-pink pigmentation (Fig. 1c). Fundoscopic exam was unremarkable.

She underwent surgical excision and debulking of the mass and cryotherapy of the surrounding conjunctiva. The biopsy of the bulbar conjunctiva showed nodular amyloid deposition (Fig. 2) highlighted with Congo red stain. Immunohistochemis-

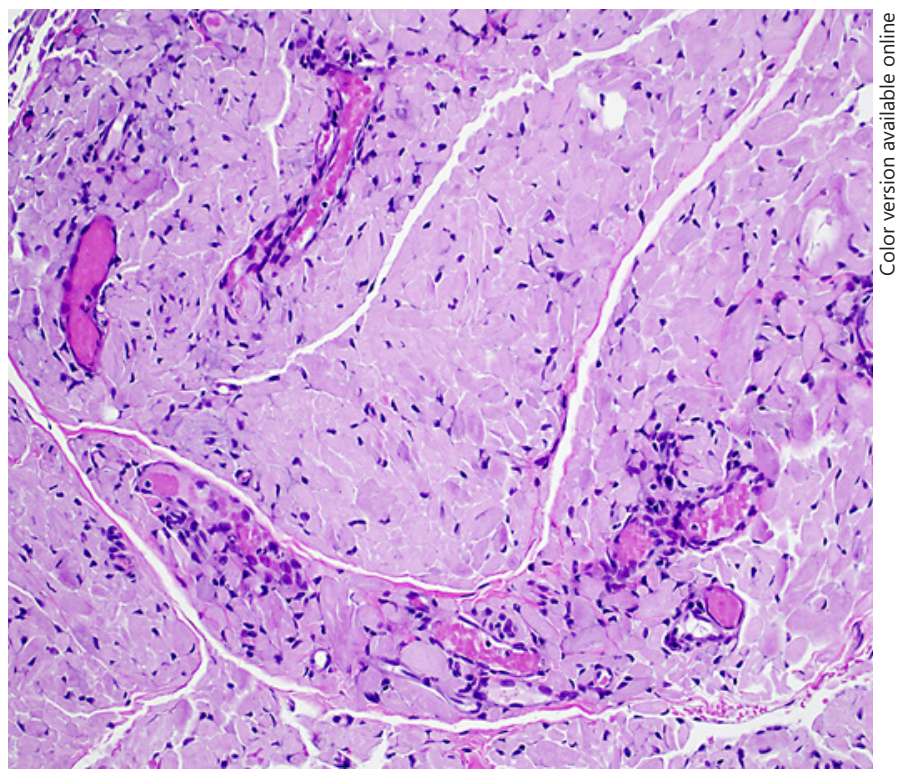

Fig. 2. HE staining of the conjunctiva showing diffuse hyaline material and small vessels classically seen in amyloid.

try demonstrated excess kappa light-chain deposition and lightchain-restricted plasma cells. Due to concern for a systemic plasma cell disorder, she underwent fine needle aspiration of abdominal fat pad, which was negative for malignant cells or amyloid on Congo red stain. However, electron microscopy showed small fragments of fibrofatty tissue with focal fibrillar deposits consistent with early amyloid. Systemic workup was notable for elevated serum free kappa light chains. Other studies (urine immunofixation, urine protein electrophoresis, and blood immunofixation) have been negative to date. Furthermore, a PET scan showed no fluorodeoxyglucose-avid lesions. The lesion has been stable for 4 months, and she continues to be followed closely.

\section{Case 4}

A 70-year-old woman presented with bilateral, progressively enlarging, hemorrhagic lower conjunctival lesions. Examination of the left eye showed smooth, hypervascular, salmon-colored masses at the medial bulbar conjunctiva and inferior fornix extending to the bulbar and palpebral conjunctiva (Fig. 1d). A smaller yellow-pinkish conjunctival mass was detected on the central lower fornix of the right eye. Visual acuity was 20/25 in each eye, intraocular pressure was $13 \mathrm{~mm} \mathrm{Hg}$ bilaterally, and extraocular movements were full. Fundoscopic exam was normal. She had no past medical history or ocular history. She underwent excision and histopathological diagnosis of conjunctival amyloidosis. She was treated with fluorometholone eye drops, and the lesions were followed for 15 years without progression. Systemic workup for rheumatologic disorders at an outside hospital was negative. 


\section{Discussion}

This case series describes 4 different cases of conjunctival amyloidosis treated at a single institution. Although commonly seen in middle-aged individuals, amyloidosis can also be found in children and adults. The average age of our patients was 41.5 years (range 11-70) at the time of diagnosis of conjunctival amyloidosis. All patients were diagnosed by biopsy, which showed $\mathrm{H}$ and $\mathrm{E}$ hyaline deposits and apple birefringence with Congo red stain. Biopsy results from 3 patients also described deposition of either kappa or lambda monoclonal immunoglobulin light chains, consistent with amyloid local (AL) type amyloidosis. (This information was not available for case 4.) Treatment included complete excision when possible and cryotherapy to reduce the cellular component of the residual infiltrate. Patients were observed for an average of 6.1 years (range 4 months to 15 years) without evidence of progression. Workup for systemic involvement was negative for all but 1 patient who was found to have signs of early systemic amyloidosis after undergoing an abdominal fat pad biopsy.

Amyloidosis can be systemic (multiple organs or tissues) or localized ( 1 site or organ). The 2 most common forms of systemic amyloidosis include immunoglobulin AL amyloidosis and AA amyloidosis. AL amyloidosis is a benign, low-grade, monoclonal light-chain gammopathy that can involve various organs and tissues [3]. This may be associated with plasma cell dyscrasias (monoclonal gammopathy of undetermined significance, multiple myeloma, and Waldenström macroglobulinemia). Reactive systemic AA amyloidosis, on the other hand, is commonly seen in patients with long-standing inflammation (e.g., rheumatologic diseases), infection, and autoinflammatory disorders due to overproduction of acute phase reactant serum AA protein, which can lead to deposits in various tissues [2]. More than 30 different proteins have been found to form amyloid fibrils in humans [1].

Local amyloidosis is most commonly caused by deposition of monoclonal immunoglobulin light chains (kappa or lambda) by a benign B-cell or plasma cell clone seen in AL amyloidosis [17]. The reason for localized deposition is unknown, but it is hypothesized to come from local synthesis of protein rather than deposition of light chains produced elsewhere [12]. Although conjunctival amyloidosis is most commonly localized, ocular involvement may represent the first sign of systemic disease [18]. Systemic amyloidosis commonly affects cardiac, liver, kidney, and splenic function. Thus, patients should be referred to an internist for further workup, which may include assessing for rheumatologic diseases and plasma cell dyscrasias. Furthermore, biopsies of the rectum, abdominal subcutaneous fat, or bone marrow can be performed.

The deposition of amyloid protein in ocular surfaces may be asymptomatic or result in various signs and symptoms. Those with conjunctival involvement most commonly present with unilateral swelling, irritation, and subconjunctival hemorrhage. Subconjunctival hemorrhage can be recurrent and is thought to be due to protein deposition in the walls of blood vessels. The most common site of involvement is the lower bulbar conjunctiva. However, eyelids, lacrimal glands, and extraocular muscles may also be involved $[3,21,22]$. Patients can also present with proptosis or ectropion requiring surgical correction.

The rarity of this disease and the variety in presenting symptoms can make a diagnosis difficult. Therefore, surgical excision and histopathological examination of biopsy specimens is critical. Congo red stain of biopsy specimens can show apple-green birefringence under polarized light, which is pathognomonic for amyloidosis. Furthermore, specimens can show proliferation of B cells and immunoglobulin light chains, which is consistent with localized AL amyloidosis. This disease is commonly localized, but patients should undergo workup to rule out systemic amyloidosis and hematological malignancies, as described above.

Treatment of conjunctival amyloidosis depends on the extent of the disease, symptoms, and the patient's general health. Often, the lesion can be observed if it does not cause significant morbidity [17]. However, patients with symptomatic disease are treated with surgical excision [23], radiotherapy, and cryotherapy [24]. Surgical debulking is the mainstay of treatment for patients with symptomatic disease, as seen in our patient cohort. However, even with surgery, patients may continue to have recurrent episodes requiring subsequent debulking and cryotherapy.

This case series highlights the various clinical presentations of conjunctival amyloidosis and the utility of surgical excision in the diagnosis and treatment of these lesions. Systemic workup of conjunctival amyloidosis should be performed and tailored to the patient's characteristics (age, gender, and ethnicity). This report suggests that, while some patients have recurrent, difficultto-treat symptoms, most patients with conjunctival amyloidosis have stable disease that can be managed with local excision followed by observation. 


\section{Statement of Ethics}

Patient consent was not necessary as this report does not contain any personal information that could lead to the identification of the patient.

\section{Disclosure Statement}

The authors have no financial interests, conflicts of interests, or disclosures related to this study.

\section{References}

1 Wechalekar AD, Gillmore JD, Hawkins PN: Systemic amyloidosis. Lancet 2016;387:26412654.

2 Hazenberg BP, van Gameren II, Bijzet J, Jager PL, van Rijswijk MH: Diagnostic and therapeutic approach of systemic amyloidosis. Neth J Med 2004;62:121-128.

3 Leibovitch I, Selva D, Goldberg RA, Sullivan TJ, Saeed P, Davis G, McCann JD, McNab A, Rootman J: Periocular and orbital amyloidosis: clinical characteristics, management, and outcome. Ophthalmology 2006;113:16571664.

4 Suesskind D, Ziemssen F, Rohrbach JM: Conjunctival amyloidosis - clinical and histopathologic features. Graefes Arch Clin Exp Ophthalmol 2015;253:1377-1383.

5 Mora-Horna ER, Rojas-Padilla R, Lopez VG, Guzman MJ, Ceriotto A, Salcedo G: Ocular adnexal and orbital amyloidosis: a case series and literature review. Int Ophthalmol 2016; 36:281-298.

6 Lee HM, Naor J, DeAngelis D, Rootman DS: Primary localized conjunctival amyloidosis presenting with recurrence of subconjunctival hemorrhage. Am J Ophthalmol 2000;129: 245-247.

7 Meduri A, Rechichi M, Mazzotta C, Scalinci SZ, Jaroudi MO: Ectropion and conjunctival mass in a patient with primary bilateral conjunctival amyloidosis. Case Rep Ophthalmol Med 2016;2016:5610753.
8 Kamal S, Goel R, Bodh SA, Madhu: Primary localized amyloidosis presenting as a tarsal mass: report of two cases. Middle East Afr J Ophthalmol 2012;19:426-428.

9 Jaswal TS, Singh S, Gupta V, Kalra R, Srivastava $\mathrm{M}$, Arora B: Isolated conjunctival amyloidosis - a case report. Indian J Pathol Microbiol 2003;46:235-236.

10 Cheong-Leen R: Primary localised conjunctival amyloidosis presenting as subconjunctival haemorrhage. Eye (Lond) 2001;15:679-680.

11 Chaturvedi P, Lala M, Desai S, Sanghvi V: A rare case of both eyelids swelling: isolated conjunctival amyloidosis. Indian J Ophthalmol 2000;48:56-57.

12 Biewend ML, Menke DM, Calamia KT: The spectrum of localized amyloidosis: a case series of 20 patients and review of the literature. Amyloid 2006;13:135-142.

13 Ali Z, Fernando B: A rare case of amyloidosis of the eyelid and conjunctiva. Case Rep Ophthalmol Med 2016;2016:2915196.

14 Aryasit O, Preechawai P, Kayasut K: Clinical presentation, treatment, and prognosis of periocular and orbital amyloidosis in a university-based referral center. Clin Ophthalmol 2013;7:801-805.

15 Abdallah AO, Westfall C, Brown H, Muzaffar J, Atrash S, Nair B: Unilateral conjunctival AL kappa amyloidosis with trace evidence of systemic amyloidosis. Am J Case Rep 2012;13: 102-105.

16 Eneh AA, Farmer J, Kratky V: Primary localized orbital amyloid: case report and literature review; 2004-2015. Can J Ophthalmol 2016;51:e131-e136.
17 Hamill EB, Thyparampil PJ, Yen MT: Localized immunoglobulin light chain amyloid of the conjunctiva confirmed by mass spectrometry without evidence of systemic disease. Ophthal Plast Reconstr Surg 2017;33:e108e110.

18 Shields JA, Eagle RC, Shields CL, Green M, Singh AD: Systemic amyloidosis presenting as a mass of the conjunctival semilunar fold. Am J Ophthalmol 2000;130:523-525.

19 Demirci H, Shields CL, Eagle RC Jr, Shields JA: Conjunctival amyloidosis: report of six cases and review of the literature. Surv Ophthalmol 2006;51:419-433.

20 Correa LJ, Maccio JP, Esposito E, Monti R, Gonzalez-Castellanos ME, Paradelo M, Serra HM, Urrets-Zavalia JA: Systemic amyloidosis with bilateral conjunctival involvement: a case report. BMC Ophthalmol 2015;15:77.

21 Mahajan D, Yadava U, Khurana N, Goyal JL: Primary localised nodular amyloidosis of the eyelid and conjunctiva: a case report. Pathology 2006;38:189-190.

22 Rodrigues G, Sanghvi V, Lala M: A rare cause of unilateral upper and lower eyelid swelling: isolated conjunctival amyloidosis. Korean J Ophthalmol 2001;15:38-40.

23 Tyradellis C, Peponis V, Kulwin DR: Surgical management of recurrent localized eyelid amyloidosis. Ophthal Plast Reconstr Surg 2006; 22:308-309.

24 Fraunfelder FW: Liquid nitrogen cryotherapy for conjunctival amyloidosis. Arch Ophthalmol 2009;127:645-648. 